\title{
Generalized total Kalman filter algorithm of nonlinear dynamic errors-in-variables model with application on indoor mobile robot positioning
}

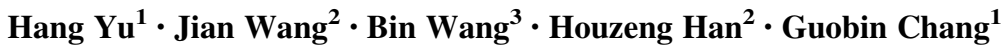

Received: 15 June 2017/ Accepted: 30 September 2017/Published online: 17 October 2017

(C) Akadémiai Kiadó 2017

\begin{abstract}
In this paper, a nonlinear dynamic errors-in-variables (DEIV) model which considers all of the random errors in both system equations and observation equations is presented. The nonlinear DEIV model is more general in the structure, which is an extension of the existing DEIV model. A generalized total Kalman filter (GTKF) algorithm that is capable of handling all of random errors in the respective equations of the nonlinear DEIV model is proposed based on the Gauss-Newton method. In addition, an approximate precision estimator of the posteriori state vector is derived. A two dimensional simulation experiment of indoor mobile robot positioning shows that the GTKF algorithm is statistically superior to the extended Kalman filter algorithm and the iterative Kalman filter (IKF) algorithm in terms of state estimation. Under the experimental conditions, the improvement rates of state variables of positions $x, y$ and azimuth $\psi$ of the GTKF algorithm are about 14,29 , and $66 \%$, respectively, compared with the IKF algorithm.
\end{abstract}

Keywords Total Kalman filter · Nonlinear dynamic errors-in-variables model · Weighted total least squares $\cdot$ Integrated navigation

Jian Wang

wjiancumt@163.com

1 School of Environment Science and Spatial Informatics, China University of Mining and Technology, Xuzhou 221116, China

2 School of Geomatics and Urban Spatial Information, Beijing University of Civil Engineering and Architecture, Beijing 100044, China

3 College of Geomatics Science and Technology, Nanjing University of Technology, Nanjing 211800, China 


\section{Introduction}

An errors-in-variables (EIV) model is different from a Gauss-Markov model, which takes the random errors of both observation vector and coefficient matrix into account. Golub and van Loan (1980) solved the EIV model by the singular value decomposition (SVD) technique, and named the algorithm as total least squares (TLS) for the first time. In the geodetic field, the TLS solution was first applied to coordinate transformation (Liu 1983; Liu and Liu 1985; Liu et al. 1987). Teunissen (1988) derived the exact solution. Thereafter, studies on the TLS theories and applications are expanded and deepened, such as weighted TLS (Schaffrin and Wieser 2008; Shen et al. 2011; Mahboub 2012; Amiri-simkooei and Jazaeri 2012; Xu et al. 2012; Fang 2013; Chang 2015; Mahboub et al. 2015; Shi et al. 2015), constrained TLS (Mahboub and Sharifi 2013; Fang 2014, 2015; Fang and Wu 2016), outliers processing for TLS (Amiri-Simkooei and Jazaeri 2013; Lu et al. 2014; Wang et al. 2016), TLS for quadratic form estimation (Fang et al. 2015), Bayesian inference for the EIV model (Fang et al. 2017), TLS prediction (Li et al. 2012; Wang et al. 2017), TLS variance component estimation (Amiri-simkooei 2013, 2016; Xu and Liu 2013, 2014; Xu 2016; Wang and Xu 2016), and TLS precision estimation (Xu et al. 2012; Amiri-Simkooei et al. 2016; Wang and Zhao 2017).

Obviously, TLS algorithms have been intensively investigated for standard (non-dynamical) EIV model. The parameter vector is time-independent within the context of the TLS principle. However, the model parameter is non-static in majority of applications. Kalman filter (KF) algorithm, which is a common algorithm to deal with dynamic model and obtain time-dependent parameters, has been widely used. It was demonstrated in numbers of studies and many different algorithms were investigated, such as the extended KF (EKF) (Gelb 1974), the iterative KF (IKF) (Bell and Cathey 1993), Sage-Husa filter (Sage and Husa 1969), the particle filter (Liu and Chen 1998), unscented KF (Julier et al. 1995), the continuous-discrete KF (Crassidis and Junkins 2011). From the viewpoints of applications, the study of KF has involved in many problems, such as integrated navigation (Han et al. 2015, 2017; Li et al. 2016), GPS positioning (Xu 2003), and target tracking (Baheti 1986).

However, the main challenge faced by the above mentioned KF algorithms is that the random errors of transition matrix of system equations and coefficient matrix of observation equations are ignored. In addition, not too many studies are found that focused on this problem. Schaffrin and Iz (2008) established the dynamic errors-in-variables (DEIV) model for the first time, and a total Kalman filter (TKF) solution within the framework of TLS principle was proposed. Considering the existence of outliers, data snooping technique have been applied to the TKF algorithm by Schaffrin and Uzun (2011). Mahboub et al. (2016) extended the TKF algorithm to a general weighted TKF (WTKF) algorithm which considers a fully correlated dispersion matrix for all of random errors of observation equations. We note that the random errors of transition matrix were not taken into account in these three literatures. Recently, Mahboub et al. (2017a, b) consider the drawback of this kind and present integrated TKF (ITKF) algorithm and constrained ITKF (CITKF) algorithm to solve the DEIV model with or without constraints. In the majority of geodetic problems, however, the elements of transition matrix and coefficient matrix are not directly measured variables but are functions of those variables. Therefore, it is necessary to study TKF algorithm under nonlinear DEIV model.

In this contribution, we proposed a generalized total Kalman filter (GTKF) algorithm for nonlinear DEIV model. Although the system equations and observation equations of the nonlinear DEIV model are structurally similar to the nonlinear Gauss-Helmert model 
(GHM) (Neitzel 2010; Chang 2015; Chang et al. 2017), the randomness of state vector is considered in this model as well. Considering the nonlinear characteristics of the nonlinear DEIV model, the derivations of the proposed algorithm fully takes advantage of the GaussNewton method of nonlinear least squares, which makes the GTKF algorithm simple in understanding and easy to implement. Then, a first order dispersion matrix of posterior state vector is obtained by using the variance propagation law. Through numerical examples, the performance of GTKF algorithm is illustrated in comparison with EKF algorithm and IKF algorithm.

The remainder of this paper is organized as follows: in Sect. 2, the nonlinear DEIV model and GTKF algorithm are formulated. In Sect. 3, the iterative scheme of GTKF algorithm is presented. Simulation experiments are illustrated and analyzed in Sect. 4. Finally in Sect. 5, conclusions are summarized.

\section{Nonlinear dynamic errors-in-variables model and its generalized total Kalman filter algorithm}

\subsection{Nonlinear dynamic errors-in-variables model}

At an epoch $k$, both system equations and observation equations are now expressed as

$$
\begin{gathered}
\boldsymbol{\xi}_{k}=\boldsymbol{\varphi}_{k}\left(\mathbf{a}_{k}-\mathbf{e}_{a_{k}}, \boldsymbol{\xi}_{k-1}\right)+\mathbf{u}_{k}, \\
\mathbf{y}_{k}=\boldsymbol{f}_{k}\left(\mathbf{b}_{k}-\mathbf{e}_{b_{k}}, \boldsymbol{\xi}_{k}\right)+\mathbf{e}_{y_{k}},
\end{gathered}
$$

where $\boldsymbol{\xi}_{k-1}$ and $\boldsymbol{\xi}_{k}$ are the $m \times 1$ time dependent state vectors (unknowns) at epoch $k-1$ and $k$, respectively, $\boldsymbol{a}_{k}$ is the $p \times 1$ observation vector contaminated by the $p \times 1$ random error vector $\boldsymbol{e}_{a_{k}}, \boldsymbol{b}_{k}$ is the $q \times 1$ observation vector contaminated by the $q \times 1$ random error vector $\boldsymbol{e}_{b_{k}}, \boldsymbol{y}_{k}$ is the $n \times 1$ vector of observations, $\boldsymbol{e}_{y_{k}}$ is the $n \times 1$ random error vector, $\boldsymbol{u}_{k}$ is the $m \times 1$ random system error vector.

The posterior estimate $\xi_{k-1}^{+}$obtained from the final result at the previous epoch $k-1$ fulfills the following equation

$$
\boldsymbol{\xi}_{k-1}^{+}=\boldsymbol{\xi}_{k-1}-\boldsymbol{w}_{k-1},
$$

where the superscript " + " represents the a posterior value, $\boldsymbol{w}_{k-1}$ is the random error of $\boldsymbol{\xi}_{k-1}^{+}$.

Assuming that all error terms in Eqs. (1), (2), and (3) are normally distributed, the stochastic model can be described as

$$
\left[\begin{array}{c}
\mathbf{w}_{k-1} \\
\mathbf{e}_{a_{k}} \\
\mathbf{u}_{k} \\
\mathbf{e}_{k}
\end{array}\right] \sim\left(\left[\begin{array}{c}
\mathbf{0} \\
\mathbf{0} \\
\mathbf{0} \\
\mathbf{0}
\end{array}\right],\left[\begin{array}{cccc}
\Sigma_{k-1} & \mathbf{0} & \mathbf{0} & \mathbf{0} \\
\mathbf{0} & \mathbf{Q}_{a_{k}} & \mathbf{0} & \mathbf{0} \\
\mathbf{0} & \mathbf{0} & \boldsymbol{\theta}_{k} & \mathbf{0} \\
\mathbf{0} & \mathbf{0} & \mathbf{0} & \mathbf{Q}_{k}
\end{array}\right]\right),
$$

where $\boldsymbol{\Sigma}_{k-1}, \boldsymbol{Q}_{a_{k}}$, and $\boldsymbol{\theta}_{k}$ are the corresponding dispersion matrices of $\boldsymbol{w}_{k-1}, \boldsymbol{e}_{a_{k}}$, and $\boldsymbol{u}_{k}, \boldsymbol{Q}_{k}$ is a $(n+q) \times(n+q)$ fully correlated dispersion matrix of $\boldsymbol{e}_{k}$, where

$$
\boldsymbol{e}_{k}=\left[\begin{array}{c}
\boldsymbol{e}_{y_{k}} \\
\boldsymbol{e}_{b_{k}}
\end{array}\right]
$$




$$
\boldsymbol{Q}_{k}=\left[\begin{array}{cc}
\boldsymbol{Q}_{y_{k}} & \boldsymbol{Q}_{y_{k} b_{k}} \\
\boldsymbol{Q}_{b_{k} y_{k}} & \boldsymbol{Q}_{b_{k}}
\end{array}\right]
$$

In Eqs. (5) and (6), $\boldsymbol{Q}_{b_{k}}$ and $\boldsymbol{Q}_{y_{k}}$ are the dispersion matrices of $\boldsymbol{e}_{b_{k}}$ and $\boldsymbol{e}_{y_{k}}, \boldsymbol{Q}_{b_{k} y_{k}}$ and $\boldsymbol{Q}_{y_{k} b_{k}}$ are the cross dispersion matrix between $\boldsymbol{e}_{b_{k}}$ and $\boldsymbol{e}_{y_{k}}$.

We further assumed that $\boldsymbol{w}_{k-1}, \boldsymbol{e}_{a_{k}}, \boldsymbol{u}_{k}$, and $\boldsymbol{e}_{k}$ are statistically independent of the current and previous state and independent of each other.

\subsection{Formulation of generalized total Kalman filter algorithm}

The standard Kalman filter algorithm can be performed through two stages, namely, the prediction and the correction. In the prediction stage, the main goal is to give the one-step prediction of the state estimate using the system equations. In the correction stage, however, the observation equations are involved to improve the current (predicted) estimate. We note that Eqs. (1) and (2) are essentially nonlinear models, the Gauss-Newton method of nonlinear least squares is employed to derive the solution of both two stages.

Since we are going to use the system equations to give the one-step prediction of the state vector $\hat{\boldsymbol{\xi}}_{k}^{-}$(the superscript " - " represents a one-step predicted value, the symbol "^ ", above a variable represents an estimate), the observation equations are not involved in the prediction stage.

The Taylor series expansion is applied to the right-hand side of Eq. (1) at the approximate values $\boldsymbol{e}_{a_{k}}^{0}$ and $\boldsymbol{\xi}_{k-1}^{+}$of $\boldsymbol{e}_{a_{k}}$ and $\boldsymbol{\xi}_{k-1}$. Thereby, Eq. (1) is now expressed as

$$
\boldsymbol{\xi}_{k}=\boldsymbol{\varphi}_{k}\left(\boldsymbol{e}_{a_{k}}^{0}, \boldsymbol{\xi}_{k-1}^{+}\right)+\mathbf{G}_{k}\left(\boldsymbol{\xi}_{k-1}-\boldsymbol{\xi}_{k-1}^{+}\right)+\mathbf{H}_{k}\left(\boldsymbol{e}_{a_{k}}-\boldsymbol{e}_{a_{k}}^{0}\right)+\boldsymbol{u}_{k},
$$

where $\mathbf{G}_{k}=\frac{\partial \boldsymbol{\varphi}_{k}\left(\mathbf{e}_{a_{k}}, \boldsymbol{\xi}_{k-1}\right)}{\partial \xi_{k-1}^{\mathrm{T}}}\left|\left(\mathbf{e}_{a_{k}}^{0}, \boldsymbol{\xi}_{k-1}^{+}\right), \mathbf{H}_{k}=\frac{\partial \boldsymbol{\varphi}_{k}\left(\mathbf{e}_{a_{k}}, \boldsymbol{\xi}_{k-1}\right)}{\partial \mathbf{e}_{a_{k}}^{\mathrm{T}}}\right|\left(\mathbf{e}_{a_{k}}^{0}, \boldsymbol{\xi}_{k-1}^{+}\right)$.

Since the prior information of $\boldsymbol{\xi}_{k-1}$ is known, the adjustment for the prediction stage can be viewed as the optimization problem with random parameters (Fang 2013; Schaffrin 2009). Therefore, the Lagrange objective function of the prediction stage can be constructed by combining Eqs. (3) and (7), namely,

$$
\begin{aligned}
\Phi_{P}= & \mathbf{w}_{k-1}^{\mathrm{T}} \boldsymbol{\Sigma}_{k-1}^{-1} \mathbf{w}_{k-1}+\mathbf{u}_{k}^{\mathrm{T}} \boldsymbol{\theta}_{k}^{-1} \mathbf{u}_{k}+\mathbf{e}_{a_{k}}^{\mathrm{T}} \mathbf{Q}_{a_{k}}^{-1} \mathbf{e}_{a_{k}}+2 \lambda_{1}^{\mathrm{T}}\left(\mathbf{w}_{k}-\boldsymbol{\xi}_{k-1}+\boldsymbol{\xi}_{k-1}^{+}\right) \\
& +2 \lambda_{2}^{\mathrm{T}}\left(\boldsymbol{\xi}_{k}-\boldsymbol{\varphi}_{k}\left(\mathbf{e}_{a_{k}}^{0}, \boldsymbol{\xi}_{k-1}^{0}\right)-\mathbf{G}_{k}\left(\boldsymbol{\xi}_{k-1}-\boldsymbol{\xi}_{k-1}^{+}\right)-\mathbf{H}_{k}\left(\mathbf{e}_{a_{k}}-\mathbf{e}_{a_{k}}^{0}\right)-\mathbf{u}_{k}\right),
\end{aligned}
$$

where $\lambda_{1}$ and $\lambda_{2}$ denote the Lagrange multipliers. The solution of the Lagrange objective function can be achieved through the following Euler-Lagrange necessary conditions

$$
\begin{gathered}
\frac{1}{2} \frac{\partial \Phi_{P}}{\partial \boldsymbol{w}_{k-1}} \mid\left(\hat{\mathbf{w}}_{k-1}^{-}, \hat{\boldsymbol{\xi}}_{k-1}, \hat{\boldsymbol{\xi}}_{k}^{-}, \hat{\mathbf{e}}_{a_{k}}^{-}, \hat{\mathbf{u}}_{k}^{-}, \hat{\boldsymbol{\lambda}}_{1}, \hat{\boldsymbol{\lambda}}_{2}\right)=\Sigma_{k-1}^{-1} \hat{\mathbf{w}}_{k-1}^{-}+\hat{\boldsymbol{\lambda}}_{1}=\mathbf{0}, \\
\frac{1}{2} \frac{\partial \Phi_{P}}{\partial \boldsymbol{\xi}_{k-1}} \mid\left(\hat{\boldsymbol{w}}_{k-1}^{-}, \hat{\boldsymbol{\xi}}_{k-1}, \hat{\boldsymbol{\xi}}_{k}^{-}, \hat{\boldsymbol{e}}_{a_{k}}^{-}, \hat{\boldsymbol{u}}_{k}^{-}, \hat{\boldsymbol{\lambda}}_{1}, \hat{\boldsymbol{\lambda}}_{2}\right)=-\hat{\boldsymbol{\lambda}}_{1}-\boldsymbol{G}_{k}^{\mathrm{T}} \hat{\boldsymbol{\lambda}}_{2}=\mathbf{0}, \\
\frac{1}{2} \frac{\partial \Phi_{P}}{\partial \boldsymbol{\xi}_{k}} \mid\left(\hat{\boldsymbol{w}}_{k-1}^{-}, \hat{\boldsymbol{\xi}}_{k-1}, \hat{\boldsymbol{\xi}}_{k}^{-}, \hat{\boldsymbol{e}}_{a_{k}}^{-}, \hat{\boldsymbol{u}}_{k}^{-}, \hat{\boldsymbol{\lambda}}_{1}, \hat{\boldsymbol{\lambda}}_{2}\right)=\hat{\boldsymbol{\lambda}}_{2}=\mathbf{0},
\end{gathered}
$$




$$
\begin{gathered}
\frac{1}{2} \frac{\partial \Phi_{P}}{\partial \boldsymbol{e}_{a_{k}}} \mid\left(\hat{\boldsymbol{w}}_{k-1}^{-}, \hat{\boldsymbol{\xi}}_{k-1}, \hat{\boldsymbol{\xi}}_{k}^{-}, \hat{\boldsymbol{e}}_{a_{k}}^{-}, \hat{\boldsymbol{u}}_{k}^{-}, \hat{\boldsymbol{\lambda}}_{1}, \hat{\boldsymbol{\lambda}}_{2}\right)=\boldsymbol{Q}_{a_{k}}^{-1} \hat{\boldsymbol{e}}_{a_{k}}^{-}-\boldsymbol{H}_{k}^{\mathrm{T}} \hat{\boldsymbol{\lambda}}_{2}=\mathbf{0}, \\
\frac{1}{2} \frac{\partial \Phi_{P}}{\partial \boldsymbol{u}_{k}} \mid\left(\hat{\boldsymbol{w}}_{k-1}^{-}, \hat{\boldsymbol{\xi}}_{k-1}, \hat{\boldsymbol{\xi}}_{k}^{-}, \hat{\boldsymbol{e}}_{a_{k}}^{-}, \hat{\boldsymbol{u}}_{k}^{-}, \hat{\boldsymbol{\lambda}}_{1}, \hat{\boldsymbol{\lambda}}_{2}\right)=\boldsymbol{\theta}_{k}^{-1} \hat{\boldsymbol{u}}_{k}^{-}-\hat{\boldsymbol{\lambda}}_{2}=\mathbf{0}, \\
\frac{1}{2} \frac{\partial \Phi_{P}}{\partial \boldsymbol{\lambda}_{1}} \mid\left(\hat{\boldsymbol{w}}_{k-1}^{-}, \hat{\boldsymbol{\xi}}_{k-1}, \hat{\boldsymbol{\xi}}_{k}^{-}, \hat{\boldsymbol{e}}_{a_{k}}^{-}, \hat{\boldsymbol{u}}_{k}^{-}, \hat{\boldsymbol{\lambda}}_{1}, \hat{\boldsymbol{\lambda}}_{2}\right)=\hat{\boldsymbol{w}}_{k-1}^{-}-\hat{\boldsymbol{\xi}}_{k-1}+\boldsymbol{\xi}_{k-1}^{+}=\mathbf{0}, \\
\frac{1}{2} \frac{\partial \Phi_{P}}{\partial \boldsymbol{\lambda}_{2}} \mid\left(\hat{\boldsymbol{w}}_{k-1}^{-}, \hat{\boldsymbol{\xi}}_{k-1}, \hat{\boldsymbol{\xi}}_{k}^{-}, \hat{\boldsymbol{e}}_{a_{k}}^{-}, \hat{\boldsymbol{u}}_{k}^{-}, \hat{\boldsymbol{\lambda}}_{1}, \hat{\boldsymbol{\lambda}}_{2}\right)=\hat{\boldsymbol{\xi}}_{k}^{-}-\boldsymbol{\varphi}_{k}\left(\boldsymbol{e}_{a_{k}}^{0}, \boldsymbol{\xi}_{k-1}^{+}\right)-\boldsymbol{G}_{k}\left(\hat{\boldsymbol{\xi}}_{k-1}-\boldsymbol{\xi}_{k-1}^{+}\right) \\
-\boldsymbol{H}_{k}\left(\hat{\boldsymbol{e}}_{a_{k}}^{-}-\boldsymbol{e}_{a_{k}}^{0}\right)-\hat{\boldsymbol{u}}_{k}^{-}=\mathbf{0}
\end{gathered}
$$

From Eqs. (9a) to (9f), we can readily obtain the following quantities of the one-step predicted values, namely,

$$
\begin{gathered}
\hat{\boldsymbol{w}}_{k-1}^{-}=\mathbf{0}, \quad \hat{\boldsymbol{\lambda}}_{1}=\mathbf{0}, \quad \hat{\lambda}_{2}=\mathbf{0}, \quad \hat{\boldsymbol{e}}_{a_{k}}^{-}=\mathbf{0}, \quad \hat{\boldsymbol{u}}_{k}^{-}=\mathbf{0}, \\
\hat{\boldsymbol{\xi}}_{k-1}=\boldsymbol{\xi}_{k-1}^{+} .
\end{gathered}
$$

By inserting Eqs. (10a) and (10b) into Eq. (9g), the one-step prediction of state vector $\xi_{k}$ is calculated as

$$
\hat{\boldsymbol{\xi}}_{k}^{-}=\boldsymbol{\varphi}_{k}\left(\boldsymbol{e}_{a_{k}}^{0}, \boldsymbol{\xi}_{k-1}^{+}\right)-\boldsymbol{H}_{k} \boldsymbol{e}_{a_{k}}^{0}
$$

We note that the second term in the right-hand side of the above formula can be omitted, since the approximate (initial) value $\boldsymbol{e}_{a_{k}}^{0}$ of $\boldsymbol{e}_{a_{k}}$ is usually set to zero for numeral calculation.

To achieve the dispersion matrix associated with $\hat{\xi}_{k}^{-}$, we form the expression for the prior error vector (Brown and Hwang, 2012)

$$
\begin{aligned}
\boldsymbol{e}_{\xi_{k}}^{-} & =\boldsymbol{\xi}_{k}-\hat{\boldsymbol{\xi}}_{k}^{-} \\
& \approx \boldsymbol{\varphi}_{k}\left(\boldsymbol{e}_{a_{k}}^{0}, \boldsymbol{\xi}_{k-1}^{+}\right)+\boldsymbol{G}_{k} \Delta \boldsymbol{\xi}_{k-1}+\boldsymbol{H}_{k}\left(\boldsymbol{e}_{a_{k}}-\boldsymbol{e}_{a_{k}}^{0}\right)+\boldsymbol{u}_{k}-\left(\boldsymbol{\varphi}_{k}\left(\boldsymbol{e}_{a_{k}}^{0}, \boldsymbol{\xi}_{k-1}^{+}\right)-\boldsymbol{H}_{k} \boldsymbol{e}_{a_{k}}^{0}\right) \\
& =\boldsymbol{G}_{k} \Delta \boldsymbol{\xi}_{k-1}+\boldsymbol{H}_{k} \boldsymbol{e}_{a_{k}}+\boldsymbol{u}_{k} \\
& =\boldsymbol{G}_{k} \boldsymbol{w}_{k-1}+\boldsymbol{H}_{k} \boldsymbol{e}_{a_{k}}+\boldsymbol{u}_{k}
\end{aligned}
$$

By using the variance propagation law to Eq. (12), the dispersion matrix of the state estimation error prior to the measurement update is as follow

$$
\mathbf{Q}_{\hat{\xi}_{k}^{-}}=\mathbf{G}_{k} \Sigma_{k-1} \mathbf{G}_{k}^{\mathrm{T}}+\mathbf{H}_{k} \mathbf{Q}_{a_{k}} \mathbf{H}_{k}^{\mathrm{T}}+\boldsymbol{\theta}_{k}
$$

Equations (11) and (13) provide the prior estimate for the correction stage. It should be pointed out that the dispersion matrix of $\hat{\xi}_{k}^{-}$is in fact a first order approximate matrix which should be updated through iterative process (Amiri-Simkooei et al. 2016; Wang et al. 2017).

In similar way, the right-hand members of Eq. (2) is expressed through Taylor series expansion at $\left(\boldsymbol{e}_{b_{k}}^{0}, \hat{\boldsymbol{\xi}}_{k}^{0}\right)$ 


$$
\mathbf{y}_{k}-\mathbf{e}_{y_{k}}=\boldsymbol{f}_{k}\left(\mathbf{e}_{b_{k}}^{0}, \hat{\xi}_{k}^{0}\right)+\mathbf{A}_{k}\left(\xi_{k}-\hat{\xi}_{k}^{0}\right)+\mathbf{B}_{k}\left(\mathbf{e}_{b_{k}}-\mathbf{e}_{b_{k}}^{0}\right),
$$

where $\mathbf{A}_{k}=\frac{\partial \boldsymbol{f}_{k}\left(\mathbf{e}_{b_{k}}, \xi_{k}\right)}{\partial \xi_{k}^{\mathrm{T}}}\left|\left(\mathbf{e}_{b_{k}}^{0}, \hat{\boldsymbol{\xi}}_{k}^{0}\right), \mathbf{B}_{k}=\frac{\partial \boldsymbol{f}_{k}\left(\mathbf{e}_{b_{k}}, \xi_{k}\right)}{\partial \mathbf{e}_{b_{k}}^{\mathrm{T}}}\right|\left(\mathbf{e}_{b_{k}}^{0}, \hat{\xi}_{k}^{0}\right)$.

Regarding the one-step prediction $\hat{\xi}_{k}^{-}$as the prior expectation of $\xi_{k}$ and taking Eq. (12) into consideration, we can formulate the objective function of the correction stage, namely,

$$
\begin{aligned}
\Phi_{C}= & \left(\mathbf{e}_{\xi_{k}}^{-}\right)^{\mathrm{T}} \mathbf{Q}_{\xi_{k}^{-}}^{-1} \mathbf{e}_{\xi_{k}}^{-}+\mathbf{e}_{k}^{\mathrm{T}} \mathbf{Q}_{k}^{-1} \mathbf{e}_{k}+2 \boldsymbol{K}_{1}^{\mathrm{T}}\left(\mathbf{e}_{\xi_{k}}^{-}-\xi_{k}+\hat{\xi}_{k}^{-}\right) \\
& +2 \boldsymbol{K}_{2}^{\mathrm{T}}\left(\mathbf{y}_{k}-\mathbf{e}_{y_{k}}-\boldsymbol{f}_{k}\left(\mathbf{e}_{b_{k}}^{0}, \hat{\boldsymbol{\xi}}_{k}^{0}\right)-\mathbf{A}_{k}\left(\xi_{k}-\hat{\xi}_{k}^{0}\right)-\mathbf{B}_{k}\left(\mathbf{e}_{b_{k}}-\mathbf{e}_{b_{k}}^{0}\right)\right),
\end{aligned}
$$

where $\boldsymbol{K}_{1}$ and $\boldsymbol{K}_{2}$ denote the Lagrange multipliers.

The following necessary conditions must hold for the purpose of optimization,

$$
\begin{gathered}
\frac{1}{2} \frac{\partial \Phi_{C}}{\partial \boldsymbol{e}_{\xi_{k}}^{-}} \mid\left(\hat{\boldsymbol{e}}_{\xi_{k}}^{-}, \hat{\boldsymbol{\xi}}_{k}^{+}, \hat{\boldsymbol{e}}_{k}, \hat{\boldsymbol{K}}_{1}, \hat{\boldsymbol{K}}_{2}\right)=\boldsymbol{Q}_{\hat{\xi}_{k}^{-}}^{-1} \hat{\boldsymbol{e}}_{\xi_{k}}^{-}+\hat{\boldsymbol{K}}_{1}=\mathbf{0}, \\
\frac{1}{2} \frac{\partial \Phi_{C}}{\partial \boldsymbol{\xi}_{k}} \mid\left(\hat{\boldsymbol{e}}_{\xi_{k}}^{-}, \hat{\boldsymbol{\xi}}_{k}^{+}, \hat{\boldsymbol{e}}_{k}, \hat{\boldsymbol{K}}_{1}, \hat{\boldsymbol{K}}_{2}\right)=-\hat{\boldsymbol{K}}_{1}-\boldsymbol{A}_{k}^{\mathrm{T}} \hat{\boldsymbol{K}}_{2}=\mathbf{0}, \\
\frac{1}{2} \frac{\partial \Phi_{C}}{\partial \boldsymbol{e}_{k}} \mid\left(\hat{\boldsymbol{e}}_{\xi_{k}}^{-}, \hat{\boldsymbol{\xi}}_{k}^{+}, \hat{\boldsymbol{e}}_{k}, \hat{\boldsymbol{K}}_{1}, \hat{\boldsymbol{K}}_{2}\right)=\boldsymbol{Q}_{k}^{-1} \hat{\boldsymbol{e}}_{k}-\left[\begin{array}{c}
\hat{\boldsymbol{K}}_{2} \\
\boldsymbol{B}_{k}^{\mathrm{T}} \hat{\boldsymbol{K}}_{2}
\end{array}\right]=\mathbf{0}, \\
\frac{1}{2} \frac{\partial \Phi_{C}}{\partial \boldsymbol{K}_{1}} \mid\left(\hat{\boldsymbol{e}}_{\xi_{k}}^{-}, \hat{\boldsymbol{\xi}}_{k}^{+}, \hat{\boldsymbol{e}}_{k}, \hat{\boldsymbol{K}}_{1}, \hat{\boldsymbol{K}}_{2}\right)=\hat{\boldsymbol{e}}_{\xi_{k}}^{-}-\hat{\boldsymbol{\xi}}_{k}^{+}+\hat{\boldsymbol{\xi}}_{k}^{-}=\mathbf{0}, \\
\frac{\partial \Phi_{C}}{2 \boldsymbol{K}_{2}} \mid\left(\hat{\boldsymbol{e}}_{\xi_{k}}^{-}, \hat{\boldsymbol{\xi}}_{k}^{+}, \hat{\boldsymbol{e}}_{k}, \hat{\boldsymbol{K}}_{1}, \hat{\boldsymbol{K}}_{2}\right)=\boldsymbol{y}_{k}-\hat{\boldsymbol{e}}_{y_{k}}-\boldsymbol{f}_{k}\left(\boldsymbol{e}_{b_{k}}^{0}, \hat{\boldsymbol{\xi}}_{k}^{0}\right) \\
-\boldsymbol{A}_{k}\left(\hat{\boldsymbol{\xi}}_{k}^{+}-\hat{\boldsymbol{\xi}}_{k}^{0}\right)-\boldsymbol{B}_{k}\left(\hat{\boldsymbol{e}}_{b_{k}}-\boldsymbol{e}_{b_{k}}^{0}\right)=\mathbf{0}
\end{gathered}
$$

From Eq. (16c), one obtains

$$
\hat{\boldsymbol{e}}_{k}=\boldsymbol{Q}_{k}\left[\begin{array}{c}
\boldsymbol{I}_{n} \\
\boldsymbol{B}_{k}^{\mathrm{T}}
\end{array}\right] \hat{\boldsymbol{K}}_{2}
$$

By inserting Eq. (17) into Eq. (16e), we have

$$
\boldsymbol{y}_{k}-\boldsymbol{f}_{k}\left(\boldsymbol{e}_{b_{k}}^{0}, \hat{\boldsymbol{\xi}}_{k}^{0}\right)-\boldsymbol{A}_{k}\left(\hat{\boldsymbol{\xi}}_{k}^{+}-\hat{\boldsymbol{\xi}}_{k}^{0}\right)+\boldsymbol{B}_{k} \boldsymbol{e}_{b_{k}}^{0}-\left[\begin{array}{ll}
\boldsymbol{I}_{n} & \boldsymbol{B}_{k}
\end{array}\right] \boldsymbol{Q}_{k}\left[\begin{array}{c}
\boldsymbol{I}_{n} \\
\boldsymbol{B}_{k}^{\mathrm{T}}
\end{array}\right] \hat{\boldsymbol{K}}_{2}=\mathbf{0} .
$$

Therefore, the Lagrange multiplier $\hat{\boldsymbol{K}}_{2}$ can be calculated as below

$$
\hat{\boldsymbol{K}}_{2}=\boldsymbol{Q}_{\eta_{k}}^{-1}\left(\boldsymbol{y}_{k}-\boldsymbol{f}_{k}\left(\boldsymbol{e}_{b_{k}}^{0}, \hat{\boldsymbol{\xi}}_{k}^{0}\right)-\boldsymbol{A}_{k}\left(\hat{\boldsymbol{\xi}}_{k}^{+}-\hat{\boldsymbol{\xi}}_{k}^{0}\right)+\boldsymbol{B}_{k} \boldsymbol{e}_{b_{k}}^{0}\right),
$$

with $\boldsymbol{Q}_{\eta_{k}}=\left[\begin{array}{ll}\boldsymbol{I}_{n} & \boldsymbol{B}_{k}\end{array}\right] \boldsymbol{Q}_{k}\left[\begin{array}{c}\boldsymbol{I}_{n} \\ \boldsymbol{B}_{k}^{\mathrm{T}}\end{array}\right]=\boldsymbol{Q}_{y_{k}}+\boldsymbol{B}_{k} \boldsymbol{Q}_{b_{k} y_{k}}+\boldsymbol{Q}_{y_{k} b_{k}} \boldsymbol{B}_{k}^{\mathrm{T}}+\boldsymbol{B}_{k} \boldsymbol{Q}_{b_{k}} \boldsymbol{B}_{k}^{\mathrm{T}}$.

Furthermore, $\hat{\boldsymbol{K}}_{1}$ can be derived by inserting Eq. (19) into Eq. (16b), namely,

$$
\hat{\boldsymbol{K}}_{1}=-\boldsymbol{A}_{k}^{\mathrm{T}} \boldsymbol{Q}_{\eta_{k}}^{-1}\left(\boldsymbol{y}_{k}-\boldsymbol{f}_{k}\left(\boldsymbol{e}_{b_{k}}^{0}, \hat{\boldsymbol{\xi}}_{k}^{0}\right)-\boldsymbol{A}_{k}\left(\hat{\boldsymbol{\xi}}_{k}^{+}-\hat{\boldsymbol{\xi}}_{k}^{0}\right)+\boldsymbol{B}_{k} \boldsymbol{e}_{b_{k}}^{0}\right) .
$$


By taking Eqs. (16a), (16d), and (20) into consideration, we have the following normal equation

$$
\left(\boldsymbol{Q}_{\hat{\xi}_{k}^{-}}^{-1}+\boldsymbol{A}_{k}^{\mathrm{T}} \boldsymbol{Q}_{\eta_{k}}^{-1} \boldsymbol{A}_{k}\right) \hat{\boldsymbol{\xi}}_{k}^{+}=\boldsymbol{Q}_{\hat{\xi}_{k}^{-}}^{-1} \hat{\boldsymbol{\xi}}_{k}^{-}+\boldsymbol{A}_{k}^{\mathrm{T}} \boldsymbol{Q}_{\eta_{k}}^{-1}\left(\boldsymbol{y}_{k}-\boldsymbol{f}_{k}\left(\boldsymbol{e}_{b_{k}}^{0}, \hat{\boldsymbol{\xi}}_{k}^{0}\right)+\boldsymbol{A}_{k} \hat{\boldsymbol{\xi}}_{k}^{0}+\boldsymbol{B}_{k} \boldsymbol{e}_{b_{k}}^{0}\right)
$$

Then the posterior estimate $\hat{\xi}_{k}^{+}$can be calculated as

$$
\hat{\boldsymbol{\xi}}_{k}^{+}=\left(\boldsymbol{Q}_{\hat{\xi}_{k}^{-}}^{-1}+\boldsymbol{A}_{k}^{\mathrm{T}} \boldsymbol{Q}_{\eta_{k}}^{-1} \boldsymbol{A}_{k}\right)^{-1}\left(\boldsymbol{Q}_{\hat{\xi}_{k}^{-}}^{-1} \hat{\boldsymbol{\xi}}_{k}^{-}+\boldsymbol{A}_{k}^{\mathrm{T}} \boldsymbol{Q}_{\eta_{k}}^{-1}\left(\boldsymbol{y}_{k}-\boldsymbol{f}_{k}\left(\boldsymbol{e}_{b_{k}}^{0}, \hat{\boldsymbol{\xi}}_{k}^{0}\right)+\boldsymbol{A}_{k} \hat{\boldsymbol{\xi}}_{k}^{0}+\boldsymbol{B}_{k} \boldsymbol{e}_{b_{k}}^{0}\right)\right) \text {. }
$$

By introducing the matrix inversion lemma (Henderson and Searle 1981)

$$
(\boldsymbol{V}+\boldsymbol{C Z D})^{-1}=\boldsymbol{V}^{-1}-\boldsymbol{V}^{-1} \boldsymbol{C}\left(\boldsymbol{Z}^{-1}+\boldsymbol{D} \boldsymbol{V}^{-1} \boldsymbol{C}\right)^{-1} \boldsymbol{D} \boldsymbol{V}^{-1}
$$

to Eq. (22), an alternative expression of $\hat{\boldsymbol{\xi}}_{k}^{+}$is presented as

$$
\hat{\xi}_{k}^{+}=\hat{\xi}_{k}^{-}+\Delta \hat{\xi}_{k}
$$

where $\Delta \hat{\boldsymbol{\xi}}_{k}=\boldsymbol{Q}_{\hat{\xi}_{k}^{-}} \boldsymbol{A}_{k}^{\mathrm{T}}\left(\boldsymbol{Q}_{\eta_{k}}+\boldsymbol{A}_{k} \boldsymbol{Q}_{\hat{\xi}_{k}} \boldsymbol{A}_{k}^{\mathrm{T}}\right)^{-1}\left(\boldsymbol{y}_{k}-\boldsymbol{f}_{k}\left(\boldsymbol{e}_{b_{k}}^{0}, \hat{\boldsymbol{\xi}}_{k}^{0}\right)-\boldsymbol{A}_{k}\left(\hat{\boldsymbol{\xi}}_{k}^{-}-\hat{\boldsymbol{\xi}}_{k}^{0}\right)+\boldsymbol{B}_{k} \boldsymbol{e}_{b_{k}}^{0}\right)$.

By substituting Eq. (19) into Eq. (17) and taking Eq. (6) into consideration, one can further obtain the specific expressions of error vectors $\hat{\boldsymbol{e}}_{y_{k}}, \hat{\boldsymbol{e}}_{b_{k}}$ as follows

$$
\begin{aligned}
& \hat{\boldsymbol{e}}_{y_{k}}=\left(\boldsymbol{Q}_{y_{k}}+\boldsymbol{Q}_{y_{k} b_{k}} \boldsymbol{B}_{k}^{\mathrm{T}}\right) \boldsymbol{Q}_{\eta_{k}}^{-1}\left(\boldsymbol{y}_{k}-\boldsymbol{f}_{k}\left(\boldsymbol{e}_{b_{k}}^{0}, \hat{\boldsymbol{\xi}}_{k}^{0}\right)-\boldsymbol{A}_{k}\left(\hat{\boldsymbol{\xi}}_{k}^{+}-\hat{\boldsymbol{\xi}}_{k}^{0}\right)+\boldsymbol{B}_{k} \boldsymbol{e}_{b_{k}}^{0}\right), \\
& \hat{\boldsymbol{e}}_{b_{k}}=\left(\boldsymbol{Q}_{b_{k} y_{k}}+\mathbf{Q}_{b_{k}} \boldsymbol{B}_{k}^{\mathrm{T}}\right) \mathbf{Q}_{\eta_{k}}^{-1}\left(\boldsymbol{y}_{k}-\boldsymbol{f}_{k}\left(\boldsymbol{e}_{b_{k}}^{0}, \hat{\boldsymbol{\xi}}_{k}^{0}\right)-\boldsymbol{A}_{k}\left(\hat{\boldsymbol{\xi}}_{k}^{+}-\hat{\boldsymbol{\xi}}_{k}^{0}\right)+\boldsymbol{B}_{k} \boldsymbol{e}_{b_{k}}^{0}\right) .
\end{aligned}
$$

Applying the variance propagation law to Eq. (24), the precision estimator of $\hat{\xi}_{k}^{+}$is derived in first order approximation via

$$
\boldsymbol{Q}_{\hat{\xi}_{k}^{+}}=\boldsymbol{Q}_{\hat{\xi}_{k}^{-}}-\boldsymbol{Q}_{\hat{\xi}_{k}^{-}} \boldsymbol{A}_{k}^{\mathrm{T}}\left(\boldsymbol{Q}_{\eta_{k}}+\boldsymbol{A}_{k} \boldsymbol{Q}_{\hat{\xi}_{k}^{-}} \boldsymbol{A}_{k}^{\mathrm{T}}\right)^{-1} \boldsymbol{A}_{k} \boldsymbol{Q}_{\hat{\xi}_{k}^{-}}
$$

Substituting the posteriori estimate $\hat{\xi}_{k}^{+}$into Eq. (12), we have

$$
\left[\begin{array}{lll}
\boldsymbol{G}_{k} & \boldsymbol{H}_{k} & \boldsymbol{I}_{m}
\end{array}\right]\left[\begin{array}{c}
\hat{\boldsymbol{w}}_{k-1} \\
\hat{\boldsymbol{e}}_{a_{k}} \\
\hat{\boldsymbol{u}}_{k}
\end{array}\right]-\left(\hat{\boldsymbol{\xi}}_{k}^{+}-\hat{\boldsymbol{\xi}}_{k}^{-}\right)=\mathbf{0} .
$$

The formulation of Eq. (28) can be viewed as the conditional adjustment in least squares theory (Zhou et al. 2014). Therefore, the estimation of error vectors $\hat{\boldsymbol{w}}_{k-1}, \hat{\boldsymbol{e}}_{a_{k}}$, and $\hat{\boldsymbol{u}}_{k}$ can be estimated as

$$
\left[\begin{array}{c}
\hat{\boldsymbol{w}}_{k-1} \\
\hat{\boldsymbol{e}}_{a_{k}} \\
\hat{\boldsymbol{u}}_{k}
\end{array}\right]=\left[\begin{array}{ccc}
\boldsymbol{\Sigma}_{k-1} & \mathbf{0} & \mathbf{0} \\
\mathbf{0} & \boldsymbol{Q}_{a_{k}} & \mathbf{0} \\
\mathbf{0} & \mathbf{0} & \boldsymbol{\theta}_{k}
\end{array}\right]\left[\begin{array}{c}
\boldsymbol{G}_{k}^{\mathrm{T}} \\
\boldsymbol{H}_{k}^{\mathrm{T}} \\
\boldsymbol{I}_{m}
\end{array}\right] \boldsymbol{Q}_{\hat{\xi}_{k}^{-}}^{-1}\left(\hat{\boldsymbol{\xi}}_{k}^{+}-\hat{\boldsymbol{\xi}}_{k}^{-}\right) .
$$

To achieve the posterior state vector, the above procedure should be implemented iteratively. 


\section{Iterative scheme of generalized total Kalman filter algorithm}

Proposed algorithm for solving generalized total Kalman filter of nonlinear DEIV model is introduced as follows. Note that the superscript $i$ denotes the iterative index.

1. Epoch $k=0$ : input prior estimate $\xi_{0}^{+}$and its dispersion matrix $\Sigma_{0}$;

2. Epoch $k=k+1$ : input $\boldsymbol{a}_{k}, \boldsymbol{b}_{k}, \boldsymbol{y}_{k}, \boldsymbol{Q}_{a_{k}}, \boldsymbol{\theta}_{k}, \boldsymbol{Q}_{k}$;

3. Set iteration counter $i=0: \hat{\boldsymbol{e}}_{a_{k}}^{(i)}=\mathbf{0}, \hat{\boldsymbol{e}}_{b_{k}}^{(i)}=\mathbf{0}$;

4. One-step prediction: $\hat{\boldsymbol{\xi}}_{k}^{-}=\boldsymbol{\varphi}_{k}\left(\hat{\boldsymbol{e}}_{a_{k}}^{(i)}, \boldsymbol{\xi}_{k-1}^{+}\right)$and set $\hat{\boldsymbol{\xi}}_{k-1}^{+(0)}=\boldsymbol{\xi}_{k-1}^{+}, \hat{\boldsymbol{\xi}}_{k}^{0}=\hat{\boldsymbol{\xi}}_{k}^{+(0)}=\hat{\boldsymbol{\xi}}_{k}^{-}$;

5. Update $\boldsymbol{G}_{k}^{(i)}, \boldsymbol{H}_{k}^{(i)}, \boldsymbol{A}_{k}^{(i)}, \boldsymbol{B}_{k}^{(i)}$ at approximate values $\left(\hat{\boldsymbol{e}}_{a_{k}}^{(i)}, \hat{\boldsymbol{\xi}}_{k-1}^{+(i)}, \hat{\boldsymbol{e}}_{b_{k}}^{(i)}, \hat{\boldsymbol{\xi}}_{k}^{+(i)}\right)$;

6. Calculate:

$$
\begin{aligned}
& \boldsymbol{Q}_{\hat{\xi}_{k}^{-}}^{(i)}=\mathbf{G}_{k}^{(i)} \boldsymbol{\Sigma}_{k-1}\left(\mathbf{G}_{k}^{(i)}\right)^{\mathrm{T}}+\mathbf{H}_{k}^{(i)} \boldsymbol{Q}_{a_{k}}\left(\mathbf{H}_{k}^{(i)}\right)^{\mathrm{T}}+\boldsymbol{\theta}_{k}, \\
& \boldsymbol{Q}_{\eta_{k}}^{(i)}=\boldsymbol{Q}_{y_{k}}+\mathbf{B}_{k}^{(i)} \boldsymbol{Q}_{b_{k} y_{k}}+\boldsymbol{Q}_{y_{k} b_{k}}\left(\mathbf{B}_{k}^{(i)}\right)^{\mathrm{T}}+\mathbf{B}_{k}^{(i)} \boldsymbol{Q}_{b_{k}}\left(\mathbf{B}_{k}^{(i)}\right)^{\mathrm{T}}, \\
& \boldsymbol{l}_{k}^{(i)}=\mathbf{y}_{k}-\boldsymbol{f}_{k}\left(\hat{\boldsymbol{e}}_{b_{k}}^{(i)}, \hat{\xi}_{k}^{+(i)}\right)-\mathbf{A}_{k}^{(i)}\left(\hat{\boldsymbol{\xi}}_{k}^{-}-\hat{\xi}_{k}^{+(i)}\right)+\mathbf{B}_{k}^{(i)} \hat{\boldsymbol{e}}_{b_{k}}^{(i)}, \\
& \Delta \hat{\boldsymbol{\xi}}_{k}^{(i)}=\boldsymbol{Q}_{\hat{\xi}_{k}^{-}}^{(i)}\left(\mathbf{A}_{k}^{(i)}\right)^{\mathrm{T}}\left(\boldsymbol{Q}_{\eta_{k}}^{(i)}+\mathbf{A}_{k}^{(i)} \boldsymbol{Q}_{\hat{\xi}_{k}^{-}}^{(i)}\left(\mathbf{A}_{k}^{(i)}\right)^{\mathrm{T}}\right)^{-1} \boldsymbol{l}_{k}^{(i)}, \\
& \hat{\xi}_{k}^{+(i+1)}=\hat{\xi}_{k}^{-}+\Delta \hat{\xi}_{k}^{(i)}, \\
& \hat{\boldsymbol{e}}_{a_{k}}^{(i+1)}=\boldsymbol{Q}_{a_{k}}\left(\mathbf{H}_{k}^{(i)}\right)^{\mathrm{T}}\left(\boldsymbol{Q}_{\hat{\xi}_{k}^{-}}^{(i)}\right)^{-1}\left(\hat{\boldsymbol{\xi}}_{k}^{+(i+1)}-\hat{\boldsymbol{\xi}}_{k}^{-}\right), \\
& \hat{\mathbf{w}}_{k-1}^{(i+1)}=\boldsymbol{\Sigma}_{k-1}\left(\mathbf{G}_{k}^{(i)}\right)^{\mathrm{T}}\left(\mathbf{Q}_{\hat{\xi}_{k}^{-}}^{(i)}\right)^{-1}\left(\hat{\boldsymbol{\xi}}_{k}^{+(i+1)}-\hat{\boldsymbol{\xi}}_{k}^{-}\right), \\
& \hat{\boldsymbol{e}}_{b_{k}}^{(i+1)}=\left(\boldsymbol{Q}_{b_{k} y_{k}}+\boldsymbol{Q}_{b_{k}}\left(\mathbf{B}_{k}^{(i)}\right)^{\mathrm{T}}\right)\left(\boldsymbol{Q}_{\eta_{k}}^{(i)}\right)^{-1} \boldsymbol{l}_{k}^{(i)}, \\
& \hat{\xi}_{k-1}^{+(i+1)}=\boldsymbol{\xi}_{k-1}^{+}+\hat{\mathbf{w}}_{k-1}^{(i+1)}, \\
& i=i+1 \text {; }
\end{aligned}
$$

7. Repeat step 5-6 until $\left\|\Delta \hat{\xi}_{k}^{(i)}-\Delta \hat{\xi}_{k}^{(i-1)}\right\|<\varepsilon$ ( $\varepsilon$ is a predefined threshold);

8. Calculate: $\boldsymbol{Q}_{\hat{\xi}_{k}^{+}}=\boldsymbol{Q}_{\hat{\xi}_{k}^{-}}^{(i)}-\boldsymbol{Q}_{\hat{\xi}_{k}^{-}}^{(i)}\left(\boldsymbol{A}_{k}^{(i)}\right)^{\mathrm{T}}\left(\boldsymbol{Q}_{\eta_{k}}^{(i)}+\boldsymbol{A}_{k}^{(i)} \boldsymbol{Q}_{\hat{\xi}_{k}^{-}}^{(i)}\left(\boldsymbol{A}_{k}^{(i)}\right)^{\mathrm{T}}\right)^{-1} \boldsymbol{A}_{k}^{(i)} \boldsymbol{Q}_{\hat{\xi}_{k}^{-}}^{(i)} ;$ 
9. For the next epoch: set $\boldsymbol{\xi}_{k}^{+}=\hat{\boldsymbol{\xi}}_{k}^{+(i)}$ and $\boldsymbol{\Sigma}_{k}=\boldsymbol{Q}_{\hat{\xi}_{k}^{+}}$;

10. If $k \leq t$ ( $t$ denotes the final epoch) return to step 2, else go to step 11;

11. End.

\section{Numerical examples and analyses}

Integrated navigation is one of the important implementation techniques for the application of indoor and outdoor positioning. For a moving unit, integrated navigation tries to determine its instantaneous position and attitude by using Kalman filter algorithm. In an outdoor environment, GPS is undoubtedly the primary choice to be implemented due to the global coverage. However, the GPS performance largely suffers from the constraint environments such as urban canyons, multipath error, and large residual atmospheric error. Therefore, it makes the carrier phase ambiguity resolution difficult to achieve and furthermore affects the high-accuracy positioning solutions (Han et al. 2017). In regards to an indoor environment, the GPS signal is blocked. Ultra-wideband (UWB) wireless radio system, which operates in the frequency band 3.1-10.6 Hz, has been the focus of attention especially in a closed environment owing to the capacity of strong anti-jamming performance and immunity of multipath. Nevertheless, the drawback of GPS and UWB is that they are vulnerable to the external environment, such as non-line of sight factor, which makes the two systems cannot be expected to achieve $100 \%$ coverage sometimes ( $\mathrm{Li}$ et al. 2016). Inertial Navigation System (INS) and other sensors, such as odometer and magnetometer, has the capability of autonomous navigation. The integration of these sensors with GPS and/or UWB can enhance the reliability and availability of an integrated system (Farrell 2008; Fan et al. 2017).

In this part, an integrated system for a mobile robot experiment in an indoor environment is introduced. Multiple sensors, such as UWB, odometer, and INS, are used to provide range, velocity and angular rate information which contribute to the determination of the final position and azimuth of the robot. Considering the moving unit is on a planar surface, then the height of it is known to be $h$ during the test, namely $z=h$. Therefore the position variable can be deemed as two dimensional, and the azimuth angle is the only attitude variable (Farrell 2008).

On the basis of the mobile robot experiment, the observation equations and the system equations are essentially nonlinear DEIV model, thus the existing TKF algorithms cannot be employed in this situation. A more reasonable result can be achieved by using the algorithm proposed in this paper.

At an epoch k, the robot kinematics is described by (Farrell 2008; Aftatah et al. 2016)

$$
\begin{aligned}
& x_{k}=x_{k-1}+\left(v_{k}-e_{v_{k}}\right) \cdot \sin \left(\psi_{k-1}+\left(\omega_{k}-e_{\omega_{k}}\right) \cdot \Delta t\right) \cdot \Delta t+u_{x}, \\
& y_{k}=y_{k-1}+\left(v_{k}-e_{v_{k}}\right) \cdot \cos \left(\psi_{k-1}+\left(\omega_{k}-e_{\omega_{k}}\right) \cdot \Delta t\right) \cdot \Delta t+u_{y}, \\
& \psi_{k}=\psi_{k-1}+\left(\omega_{k}-e_{\omega_{k}}\right) \cdot \Delta t+u_{\psi},
\end{aligned}
$$

where $\psi_{k}$ denotes the azimuth angle, $\Delta t$ denotes the sampling interval, $v_{k}$ is the velocity measured by odometer and its direction is the corresponding to the moving direction, $e_{v_{k}}$ is the error of $v_{k}, \omega_{k}$ denotes the angular rate measured by gyro during the sampling interval $\Delta t, e_{\omega_{k}}$ is the error of $\omega_{k}$, the symbols $u_{x}, u_{y}$, and $u_{\psi}$ represent system errors based on violation of the no-slip assumption.

Equation (31) has the following matrix form 


$$
\boldsymbol{\xi}_{k}=\boldsymbol{\xi}_{k-1}+\left[\begin{array}{c}
\left(v_{k}-e_{v_{k}}\right) \cdot \boldsymbol{M} \\
\omega_{k}-e_{\omega_{k}}
\end{array}\right] \cdot \Delta t+\mathbf{u}_{k}
$$

with $\boldsymbol{\xi}_{k}=\left[\begin{array}{lll}x_{k} & y_{k} & \psi_{k}\end{array}\right]^{\mathrm{T}}, \boldsymbol{M}=\left[\begin{array}{c}\sin \left(\psi_{k-1}+\left(\omega_{k}-e_{\omega_{k}}\right) \cdot \Delta t\right) \\ \cos \left(\psi_{k-1}+\left(\omega_{k}-e_{\omega_{k}}\right) \cdot \Delta t\right)\end{array}\right]$ and $\mathbf{u}_{k}=\left[\begin{array}{lll}u_{x} & u_{y} & u_{\psi}\end{array}\right]^{\mathrm{T}}$.

At epoch $\mathrm{k}$,

$$
\begin{gathered}
\boldsymbol{\varphi}_{k}=\boldsymbol{\xi}_{k-1}+\left[\begin{array}{c}
\left(v_{k}-e_{v_{k}}\right) \cdot \boldsymbol{M} \\
\omega_{k}-e_{\omega_{k}}
\end{array}\right] \cdot \Delta t, \\
\mathbf{a}_{k}=\left[\begin{array}{ll}
v_{k} & \omega_{k}
\end{array}\right]^{\mathrm{T}}, \mathbf{e}_{a_{k}}=\left[\begin{array}{ll}
e_{v_{k}} & e_{\omega_{k}}
\end{array}\right]^{\mathrm{T}} .
\end{gathered}
$$

Therefore,

$$
\mathbf{G}_{k}=\left(\frac{\partial \boldsymbol{\varphi}_{k}}{\partial \xi_{k-1}^{\mathrm{T}}}\right)^{0}=\left[\left(\frac{\partial \boldsymbol{\varphi}_{k}}{\partial x_{k-1}}\right)^{0} \quad\left(\frac{\partial \boldsymbol{\varphi}_{k}}{\partial y_{k-1}}\right)^{0} \quad\left(\frac{\partial \boldsymbol{\varphi}_{k}}{\partial \psi_{k-1}}\right)^{0}\right],
$$

with $\frac{\partial \varphi_{k}}{\partial x_{k-1}}=\left[\begin{array}{l}1 \\ 0 \\ 0\end{array}\right], \frac{\partial \varphi_{k}}{\partial y_{k-1}}=\left[\begin{array}{l}0 \\ 1 \\ 0\end{array}\right], \frac{\partial \varphi_{k}}{\partial \psi_{k-1}}=\left[\begin{array}{c}\left(v_{k-1}-e_{v_{k-1}}\right) \cdot \cos \left(\psi_{k-1}+\left(\omega_{k}-e_{\omega_{k}}\right) \cdot \Delta t\right) \\ -\left(v_{k-1}-e_{v_{k-1}}\right) \cdot \sin \left(\psi_{k-1}+\left(\omega_{k}-e_{\omega_{k}}\right) \cdot \Delta t\right) \\ 1\end{array}\right] \cdot \Delta t$.

$$
\mathbf{H}_{k}=\left(\frac{\partial \boldsymbol{\varphi}_{k}}{\partial \mathbf{e}_{a_{k}}^{T}}\right)^{0}=\left[\left(\frac{\partial \boldsymbol{\varphi}_{k}}{\partial e_{v_{k}}}\right)^{0} \quad\left(\frac{\partial \boldsymbol{\varphi}_{k}}{\partial e_{\omega_{k}}}\right)^{0}\right]
$$

where $\frac{\partial \boldsymbol{\varphi}_{k}}{\partial e_{v_{k}}}=-\left[\begin{array}{c}\boldsymbol{M} \\ 0\end{array}\right] \cdot \Delta t, \frac{\partial \boldsymbol{\varphi}_{k}}{\partial e_{\omega_{k}}}=\left[\begin{array}{c}-\left(v_{k}-e_{v_{k}}\right) \cdot \cos \left(\psi_{k-1}+\left(\omega_{k}-e_{\omega_{k}}\right) \cdot \Delta t\right) \cdot \Delta t \\ \left(v_{k}-e_{v_{k}}\right) \cdot \sin \left(\psi_{k-1}+\left(\omega_{k}-e_{\omega_{k}}\right) \cdot \Delta t\right) \cdot \Delta t \\ -1\end{array}\right] \cdot \Delta t$.

The observation equations are constructed with the range and azimuth angle information obtained from $l$ s base stations and magnetometer measurement, namely,

$$
\left[\begin{array}{c}
p_{1 k} \\
\vdots \\
p_{l k} \\
\tilde{\psi}_{k}
\end{array}\right]-\left[\begin{array}{c}
e_{p_{1 k}} \\
\vdots \\
e_{p_{l k}} \\
e_{\psi_{k}}
\end{array}\right]=\left[\begin{array}{c}
\sqrt{\left(x_{s}^{1}-e_{x_{s}^{1}}-x_{k}\right)^{2}+\left(y_{s}^{1}-e_{y_{s}^{1}}-y_{k}\right)^{2}} \\
\vdots \\
\sqrt{\left(x_{s}^{l}-e_{x_{s}^{l}}-x_{k}\right)^{2}+\left(y_{s}^{l}-e_{y_{s}^{l}}-y_{k}\right)^{2}} \\
\psi_{k}
\end{array}\right],
$$

where $p_{1 k} \cdots p_{l k}$ are the 1 range measurements from the base stations to the tested subject position, $\tilde{\psi}_{k}$ is the azimuth angle obtained from magnetometer measurement, $\left(x_{s}^{1}\right.$, $\left.y_{s}^{1}\right) \cdots\left(x_{s}^{l}, y_{s}^{l}\right)$ are the positions of base stations which are corrupted by random errors.

Thus, at epoch $\mathrm{k}$,

$$
\begin{gathered}
\boldsymbol{f}_{k}=\left[\begin{array}{c}
\sqrt{\left(x_{s}^{1}-e_{x_{s}^{1}}-x_{k}\right)^{2}+\left(y_{s}^{1}-e_{y_{s}^{1}}-y_{k}\right)^{2}} \\
\vdots \\
\sqrt{\left(x_{s}^{l}-e_{x_{s}^{l}}-x_{k}\right)^{2}+\left(y_{s}^{l}-e_{y_{s}^{l}}-y_{k}\right)^{2}} \\
\psi_{k}
\end{array}\right], \\
\mathbf{b}_{k}=\left[\begin{array}{lllll}
x_{s}^{1} & y_{s}^{1} & \cdots & x_{s}^{l} & y_{s}^{l}
\end{array}\right]^{\mathrm{T}}, \quad \mathbf{e}_{b_{k}}=\left[\begin{array}{lllll}
e_{x_{s}^{1}} & e_{y_{s}^{1}} & \cdots & e_{x_{s}^{l}} & e_{y_{s}^{l}}
\end{array}\right]^{\mathrm{T}} .
\end{gathered}
$$


The matrices $\mathbf{A}_{k}$ and $\mathbf{B}_{k}$ can be derived by

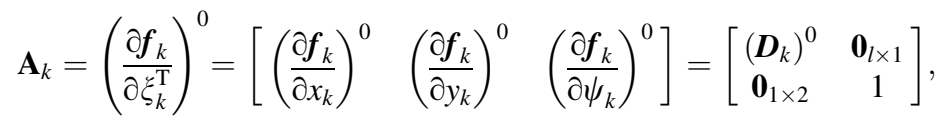

and $\quad \boldsymbol{D}_{k}=\left[\left(\boldsymbol{D}_{k}^{1}\right)^{\mathrm{T}} \cdots\left(\boldsymbol{D}_{k}^{i}\right)^{\mathrm{T}} \cdots\left(\boldsymbol{D}_{k}^{l}\right)^{\mathrm{T}}\right]^{\mathrm{T}}, \quad \boldsymbol{D}_{k}^{i}=\left[\begin{array}{ll}D_{k}^{i 1} & D_{k}^{i 2}\end{array}\right], \quad D_{k}^{i 1}=$ $-\frac{x_{s}^{i}-e_{x_{s}^{i}}-x_{k}}{\sqrt{\left(x_{s}^{i}-e_{x_{s}^{i}}-x_{k}\right)^{2}+\left(y_{s}^{i}-e_{y_{s}^{i}}-y_{k}\right)^{2}}}, D_{k}^{i 2}=-\frac{y_{s}^{i}-e_{y_{s}^{i}}-y_{k}}{\sqrt{\left(x_{s}^{i}-e_{x_{s}^{i}}-x_{k}\right)^{2}+\left(y_{s}^{i}-e_{y_{s}^{i}}-y_{k}\right)^{2}}}$.

$$
\mathbf{B}_{k}=\left(\frac{\partial \boldsymbol{f}_{k}}{\partial \mathbf{e}_{b_{k}}^{\mathrm{T}}}\right)^{0}=\left[\begin{array}{c}
\left(\boldsymbol{D}^{\prime}{ }_{k}\right)^{0} \\
\mathbf{0}_{1 \times 2 l}
\end{array}\right]
$$

with $\boldsymbol{D}_{k}^{\prime}=\left[\begin{array}{ccc}\boldsymbol{D}_{k}^{1} & \mathbf{0} & \mathbf{0} \\ \mathbf{0} & \ddots & \mathbf{0} \\ \mathbf{0} & \mathbf{0} & \boldsymbol{D}_{k}^{l}\end{array}\right]$.

The superscripts "0" in Eqs. (34), (35), (39) and (40) represent that related expressions are replaced by the corresponding approximate vectors $\left(\xi_{k-1}^{0}, \mathbf{e}_{a_{k}}^{0}\right)$ and $\left(\xi_{k}^{0}, \mathbf{e}_{b_{k}}^{0}\right)$.

Assuming that all the error vectors mentioned above are normally distributed and are generated with the given dispersion matrices.

At initial epoch, i.e. $\mathrm{k}=0$, the dispersion matrix of the random error vector of $\xi_{0}$ is set to $\Sigma_{0}=\operatorname{diag}\left(\left[1 \mathrm{~cm}^{2}, 1 \mathrm{~cm}^{2},\left(0.5^{\circ}\right)^{2}\right]\right)$. For the system equations, the variance of $e_{v_{k}}$ and $e_{\omega_{k}}$ are set to $\sigma_{e_{v}}^{2}=(0.9 \mathrm{~m} / \mathrm{s})^{2}$ and $\sigma_{e_{\omega}}^{2}=\left(0.8^{\circ} / \mathrm{s}\right)^{2}$, therefore, $\boldsymbol{Q}_{a_{k}}=\operatorname{diag}\left(\sigma_{e_{v}}^{2}, \sigma_{e_{\omega}}^{2}\right)$; the dispersion matrix of random system error vector $\boldsymbol{u}_{k}$ is set to $\boldsymbol{\theta}_{k}=\operatorname{diag}\left(\left[1 \mathrm{~cm}^{2}, 1 \mathrm{~cm}^{2},\left(0.1^{\circ}\right)^{2}\right]\right)$. For the observation equations, the true positions of base stations $\left(x_{i}^{s}, y_{i}^{s}\right)$, where $i=1,2,3,4$, are given as: $(0.5 \mathrm{~m}, 1 \mathrm{~m}),(0.5 \mathrm{~m}, 12 \mathrm{~m})$, $(6 \mathrm{~m}, 12 \mathrm{~m})$, and $(6 \mathrm{~m}, 1 \mathrm{~m})$; The true positions are biased by the random errors with the accuracy of $3 \mathrm{~cm}$; Range and azimuth measurements are biased by the random errors with the accuracy of $6 \mathrm{~cm}$ and $0.5^{\circ}$, respectively.

In this experiment, we set the refresh rate of correction stage is $1 \mathrm{~Hz}$ while the prediction stage is set to $100 \mathrm{~Hz}$, which means that the sampling interval of $\Delta t$ is $0.01 \mathrm{~s}$. It is thus clear that most of the time only predictions are computed, but corrections are made only when range measurements and azimuth angles are obtained.

In the application of GTKF algorithm, four different trajectories are simulated in an indoor environment (see Fig. 1). We note that the initial true state vector is given as $\xi_{0}=\left[\begin{array}{lll}1 \mathrm{~m} & 2 \mathrm{~m} & 30^{\circ}\end{array}\right]^{\mathrm{T}}$ for the first trajectory (i.e. Fig. 1a), for the second and third trajectories (i.e. Fig. $1 \mathrm{~b}, \mathrm{c}$ ), we set $\xi_{0}=\left[\begin{array}{lll}1 \mathrm{~m} & 2 \mathrm{~m} & 0^{\circ}\end{array}\right]^{\mathrm{T}}$, and for the last trajectory (i.e. Fig. 1d), we set $\xi_{0}=\left[\begin{array}{lll}1 \mathrm{~m} & 2 \mathrm{~m} & 60^{\circ}\end{array}\right]^{\mathrm{T}}$.

Since the algorithms proposed by Schaffrin and Iz (2008) and Mahboub et al. (2016) can only deal with linear model situation, in this section, the following three schemes are implemented and compared to show the effectiveness of the proposed GTKF algorithm.

Scheme 1 Extended Kalman filter (EKF) algorithm

Scheme 2 Iterative Kalman filter (IKF) algorithm (Bell and Cathey 1993);

Scheme 3 Generalized total Kalman filter (GTKF) algorithm presented in this paper 
The threshold for IKF and GTKF are both set to $10^{-6}$. The test results of the above three schemes according to the four simulated trajectories are illustrated in Fig. 2. As can be seen from (a-d) of Fig. 2, GTKF algorithm (Scheme 3) can achieve the best estimates, which fits the true solution line better in contrast to extended Kalman filter (Scheme 1) and iterative Kalman filter (Scheme 2) algorithms. It should be pointed out that Schemes 1 and 2 ignore the random observational errors in both system equations and observation equations. Nevertheless, GTKF (Scheme 3) algorithm takes all of random errors into consideration. We hence draw the conclusion that GTKF algorithm is more reasonable in theory.

In addition, we run the four simulation experiments based on different trajectories for 10,000 times. The statistics on the absolute errors (i.e. the estimated position and attitude variables minus their counterparts of true values) of each algorithm are presented in Fig. 3.

From (a-d) of Fig. 3, it is not difficult to find that the IKF algorithm has a slight improvement in comparison with EKF algorithm in terms of statistical absolute errors. Additionally, the absolute errors obtained by GTKF algorithm are generally smaller than those obtained by the other two algorithms. For the proposed GTKF algorithm, the $x$ variable is increased by $14 \%$, the $y$ variable is increased by $29 \%$, and the $\psi$ variable is increased by $66 \%$ in contrast to the IKF algorithm.

Here, we analyze the computational complexity in terms of the proposed GTKF algorithm. The computational complexity of EKF and GTKF is compared. It should be pointed out that the complexity mentioned here is only refers to the time complexity. Table 1 lists the computational complexity of some basic equations of the proposed GTKF algorithm.
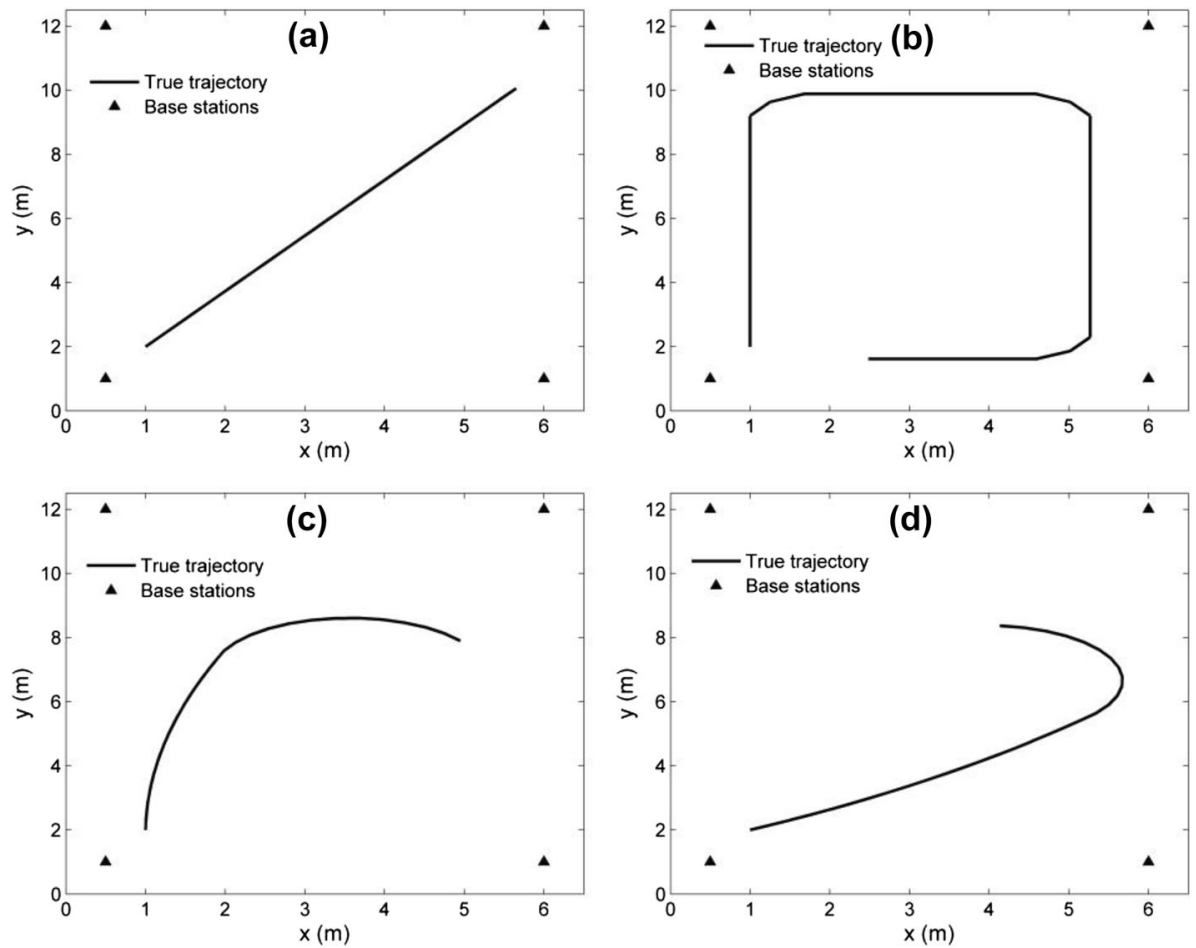

Fig. 1 The top view of the simulated trajectories 

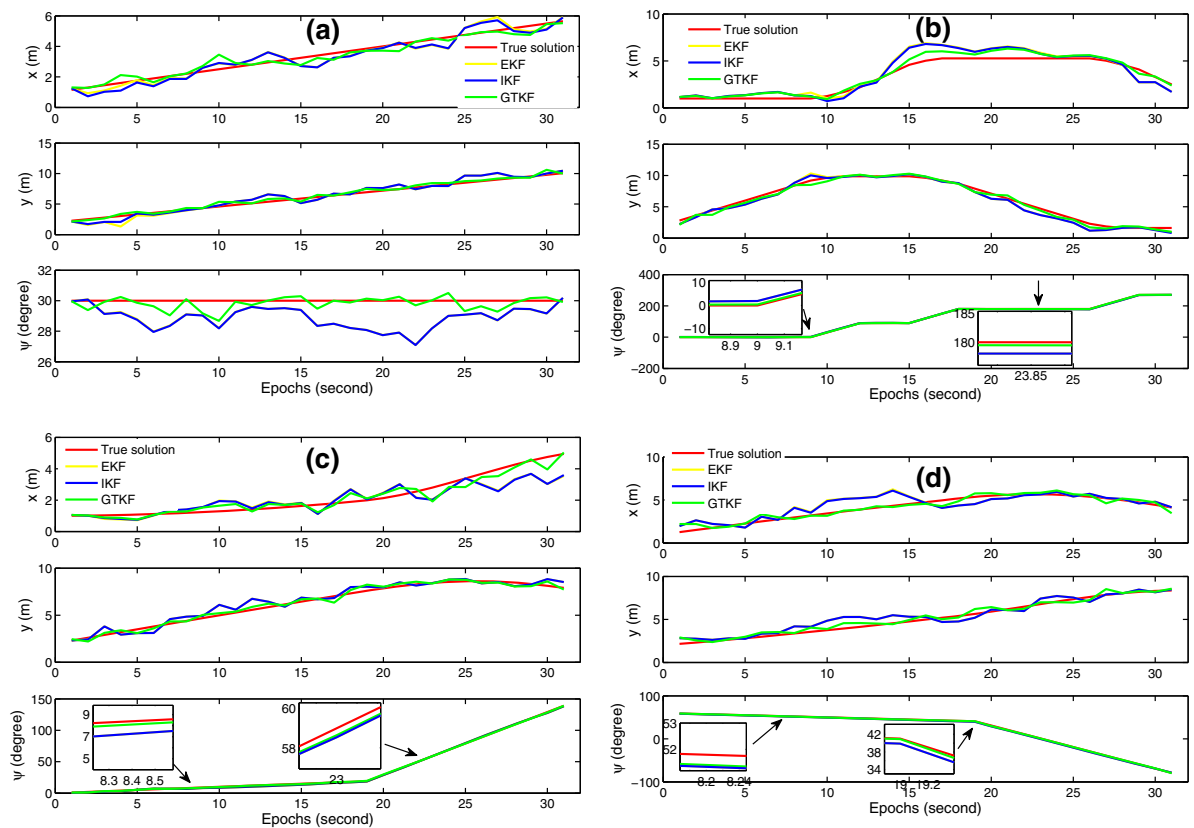

Fig. 2 The position and attitude solutions of different algorithms: a-d are the solutions based on the simulated trajectory of Fig. 1a-d, respectively

The computational complexity of the one-step prediction [i.e. Eq. (11)] is the same for both EKF and GTKF. Thus, for the sake of simplicity, the complexity of the one-step prediction is not considered. The GTKF algorithm mainly involves Eqs. (30a)-(30i) and Eq. (27). We assume that the average iteration number is $T$. Hence, according to Table 1 , the computational complexity of the GTKF algorithm is obtained as

$$
\begin{aligned}
S_{\mathrm{GTKF}}= & (8 T+2) m^{3}+(4 T+4) n^{2} m+(4 T+6) n m^{2}+(T-3) n m-(T+1) m^{2} \\
& +4 T m^{2} p+4 T m p^{2}-T m p+3 T m+8 T n^{2} q+4 T n q^{2}+2 T n q+T n-T p \\
& -T q+(2 T+1) \mathrm{O}\left(n^{3}\right)+2 T \mathrm{O}\left(m^{3}\right) .
\end{aligned}
$$

It is not hard to conclude that the computational complexity of EKF algorithm is (Merwe and Wan 2001)

$$
S_{\mathrm{EKF}}=6 m^{3}+8 n^{2} m+10 n m^{2}-2 n m-2 m^{2}+n+2 \mathrm{O}\left(n^{3}\right) .
$$

From Eqs. (41) and (42), we know that the EKF algorithm and the proposed GTKF algorithm are essentially a linear time algorithm. The computational complexities of EKF and GTKF will be proportional to $\mathrm{O}\left(\mathrm{m}^{3}\right)$ and/or $\mathrm{O}\left(n^{3}\right)$ arithmetic operations. In addition, the average iteration numbers of the GTKF algorithm is $T=6.3051$ in this example. Therefore, compared with the EKF algorithm, the complexity of the GTKF algorithm is moderate, especially when considering the modern high-performance computers.

From the iterative scheme of Sect. 3, we found that once the error terms $\mathbf{e}_{a_{k}}$ and $\mathbf{e}_{b_{k}}$ are set to zero, GTKF algorithm will be degraded to IKF algorithm. When the DEIV model is linear and the random errors of transition matrix are ignored, the proposed algorithm will 

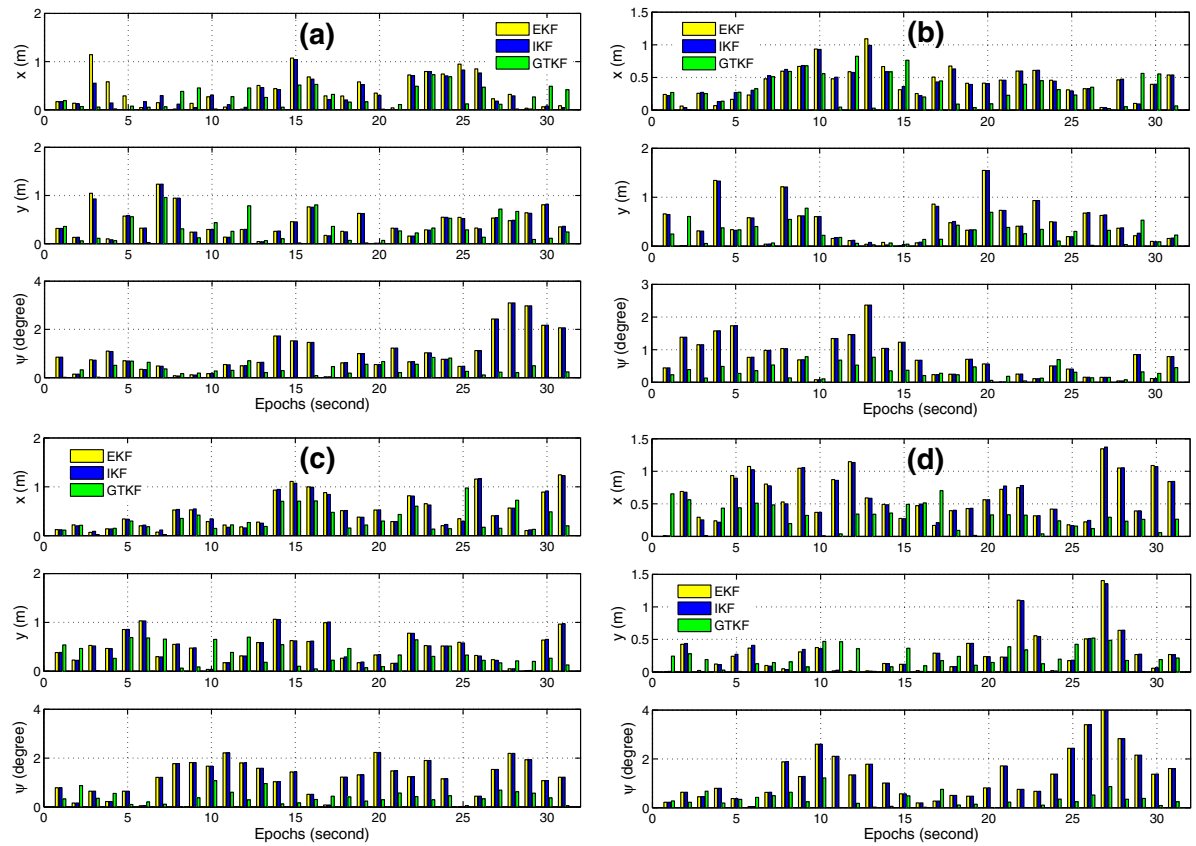

Fig. 3 The mean values of absolute error of different algorithms from 10,000 simulations: a-d are the mean values based on the simulated trajectory of Fig. 1a-d, respectively

Table 1 Computational complexities of some basic equations of GTKF

\begin{tabular}{lll}
\hline Equation & Addition and multiplication & Division and matrix inversion \\
\hline$(27)$ & $4 n^{2} m+6 n m^{2}+2 m^{3}-3 n m-m^{2}$ & $\mathrm{O}\left(n^{3}\right)$ \\
$(30 \mathrm{a})$ & $4 m^{3}+2 m^{2} p+2 m p^{2}-m^{2}-m p$ & 0 \\
$(30 \mathrm{~b})$ & $6 n^{2} q+2 n q^{2}-n q$ & 0 \\
$(30 \mathrm{c})$ & $2 n m+2 n q+n+m$ & 0 \\
$(30 \mathrm{~d})$ & $4 n^{2} m+4 n m^{2}-n m-m$ & $\mathrm{O}\left(n^{3}\right)$ \\
$(30 \mathrm{e})$ & $m$ & 0 \\
$(30 \mathrm{f})$ & $2 m^{2} p+2 m p^{2}+m-p$ & $\mathrm{O}\left(m^{3}\right)$ \\
$(30 \mathrm{~g})$ & $4 m^{3}$ & $\mathrm{O}\left(m^{3}\right)$ \\
$(30 \mathrm{~h})$ & $2 n^{2} q+2 n q^{2}+n q-q$ & $\mathrm{O}\left(n^{3}\right)$ \\
$(30 \mathrm{i})$ & $m$ & 0 \\
\hline
\end{tabular}

be numerically equivalent to the WTKF algorithm. This can be illustrated by the following underlying equations. For the sake of simplicity, the matrix and vector symbols in the right-hand side of equations of the remaining paragraph are directly abstracted from Mahboub et al. (2016). When the DEIV model appears in a linear form, the vector $\hat{\xi}_{k}^{-}$ becomes $\hat{\boldsymbol{\xi}}_{k}^{-}=\hat{\boldsymbol{x}}_{i}$, the vector $\boldsymbol{\xi}_{k}^{0}$ becomes $\boldsymbol{\xi}_{k}^{0}=\hat{\boldsymbol{x}}_{i}+\breve{\boldsymbol{\mu}}_{i}$, the matrix $\boldsymbol{A}_{k}$ becomes $\boldsymbol{A}_{k}=\boldsymbol{A}_{i}-\breve{\boldsymbol{E}}_{A_{i}}$, the symbol $\boldsymbol{B}_{k} \boldsymbol{e}_{b_{k}}^{0}$ becomes $\boldsymbol{B}_{k} \boldsymbol{e}_{b_{k}}^{0}=-\breve{\boldsymbol{E}}_{A_{i}}\left(\hat{\boldsymbol{x}}_{i}+\breve{\boldsymbol{\mu}}_{i}\right)$, hence the vector 
function $\boldsymbol{f}_{k}\left(\boldsymbol{e}_{b_{k}}^{0}, \hat{\boldsymbol{\xi}}_{k}^{0}\right)$ is represented as $\boldsymbol{f}_{k}\left(\boldsymbol{e}_{b_{k}}^{0}, \hat{\boldsymbol{\xi}}_{k}^{0}\right)=\left(\boldsymbol{A}_{i}-\breve{\boldsymbol{E}}_{A_{i}}\right)\left(\hat{\boldsymbol{x}}_{i}+\breve{\boldsymbol{\mu}}_{i}\right)$, and finally the variance-covariance $\boldsymbol{Q}_{\eta_{k}}$ becomes $\boldsymbol{Q}_{\eta_{k}}=\left(\boldsymbol{N}-\left(\left(\breve{\boldsymbol{\mu}}_{i}+\hat{\boldsymbol{x}}_{i}\right)^{\mathrm{T}} \otimes \boldsymbol{I}_{m}\right) \boldsymbol{M}\right) \boldsymbol{Q}\left(\boldsymbol{N}-\left(\left(\breve{\boldsymbol{\mu}}_{i}+\hat{\boldsymbol{x}}_{i}\right)^{\mathrm{T}} \otimes\right.\right.$ $\left.\left.\boldsymbol{I}_{m}\right) \boldsymbol{M}\right)^{\mathrm{T}}$. Moreover, if the random errors of transition matrix are omitted, $\boldsymbol{Q}_{\hat{\xi}_{k}^{-}}$will be equivalent to $\boldsymbol{Q}_{\hat{\xi}_{k}^{-}}=\boldsymbol{\theta}_{i}+\boldsymbol{\Phi}_{i} \Sigma_{i-1}^{0} \boldsymbol{\Phi}_{i}^{\mathrm{T}}$. Taking all of these equations into consideration,

Eq. (24) is in fact numerically identical to the solution $\breve{\boldsymbol{x}}_{i}=\hat{\boldsymbol{x}}_{i}+\breve{\boldsymbol{\mu}}_{i}$ in Mahboub et al. (2016). Furthermore, based on the similar analysis, GTKF algorithm is also numerically identical to TKF algorithm by further discarding the correlation of coefficient matrix and observation vector.

\section{Conclusions}

The main goal of this paper is to derive a universal Kalman filter, named generalized total Kalman filter (GTKF) algorithm, for nonlinear dynamic errors-in-variables (DEIV) model. The nonlinear DEIV model is presented which considers all of random errors in both system equations and observation equations. In addition, a GTKF algorithm and its corresponding iterative scheme are derived and designed. The derivations of the GTKF algorithm fully takes advantage of the Gauss-Newton method of nonlinear least squares, which makes the GTKF algorithm simple in understanding and easy to implement. The results of the indoor mobile robot experiments clearly show a significant improvement of GTKF algorithm in the accuracy of the estimated state vectors, and hence validate the effectiveness of the proposed algorithm. The existing TKF algorithm and WTKF algorithm can be viewed as a special form of GTKF algorithm under different assumptions. Therefore, the developed algorithm has a wider range of applications. However, the current study assumes that random errors of the nonlinear DEIV model have the same variance component and are not contaminated by gross errors. For future studies, the variance component estimation and data-snooping and/or robust estimation methods will be adopted to solve these problems.

Acknowledgements We are grateful to the editor László Bányai and two anonymous reviewers for their valuable comments. This research is supported by the National Key Research and Development Program of China (2016YFC0803103).

\section{References}

Aftatah M, Lahrech A, Abounada A, Soulhi A (2016) GPS/INS/Odometer data fusion for land vehicle localization in GPS denied environment. Mod Appl Sci 11(1):62-75

Amiri-simkooei AR (2013) Application of least squares variance component estimation to errors-in-variables models. J Geod 87:935-944

Amiri-Simkooei AR (2016) Non-negative least-squares variance component estimation with application to GPS time series. J Geod 90:451-466

Amiri-simkooei AR, Jazaeri S (2012) Weighted total least squares formulated by standard least squares theory. J Geod Sci 2:113-124

Amiri-Simkooei AR, Jazaeri S (2013) Data-snooping procedure applied to errors-in-variables models. Stud Geophys Geod 57:426-441

Amiri-Simkooei AR, Zangeneh-Nejad F, Asgari J (2016) On the covariance matrix of weighted total leastsquares estimates. J Surv Eng 142:04015014

Baheti RS (1986) Efficient approximation of Kalman filter for target tracking. IEEE Trans Aerosp Electron Syst. doi:10.1109/TAES.1986.310687 
Bell BM, Cathey FW (1993) The iterated Kalman filter update as a Gauss-Newton method. IEEE Trans Autom Control 38:294-297. doi:10.1109/9.250476

Brown RG, Hwang PYC (2012) Introduction to random signals and applied Kalman filtering: with MATLAB exercises and solutions, 4th edn. Wiley, New York

Chang GB (2015) On least-squares solution to 3D similarity transformation problem under Gauss-Helmert model. J Geod 89:573-576

Chang GB, Xu TH, Wang QX, Zhang SB, Chen GL (2017) A generalization of the analytical least-squares solution to the 3D symmetric Helmert coordinate transformation problem with an approximate error analysis. Adv Space Res 59:2600-2610

Crassidis JL, Junkins JL (2011) Optimal estimation of dynamic systems, 2nd edn. Applied mathematics and nonlinear science series. Chapman \& Hall/CRC, New York

Fan QG, Sun BW, Sun Y, Wu YH, Zhuang XP (2017) Data fusion for indoor mobile robot positioning based on tightly coupled INS/UWB. J Navig. doi:10.1017/S0373463317000194

Fang X (2013) Weighted total least squares: necessary and sufficient conditions, fixed and random parameters. J Geod 87:733-749

Fang X (2014) On non-combinatorial weighted total least squares with inequality constraints. J Geod $88: 805-816$

Fang X (2015) Weighted total least-squares with constraints: a universal formula for geodetic symmetrical transformations. J Geod 89:459-469

Fang X, Wu Y (2016) On the errors-in-variables model with equality and inequality constraints for selected numerical examples. Acta Geod Geophys 51:515-525

Fang X, Wang J, Li BF, Zeng WX, Yao YB (2015) On total least squares for quadratic form estimation. Stud Geophys Geod 59(3):366-379

Fang X, Li BF, Alkhatib H, Zeng WX, Yao YB (2017) Bayesian inference for the errors-in-variables model. Stud Geophys Geod 61(1):35-52

Farrell J (2008) Aided navigation: GPS with high rate sensors. McGraw-Hill Inc, New York

Gelb A (1974) Applied optimal estimation. MIT Press, Cambridge

Golub G, van Loan C (1980) An analysis of the total least-squares problem. SIAM J Numer Anal 17:883-893

Han HZ, Wang J, Wang JL, Tan XL (2015) Performance analysis on carrier phase-based tightly-coupled GPS/BDS/INS integration in GNSS degraded and denied environments. Sensors 15:8685-8711

Han HZ, Wang J, Wang JL, Hernandez A (2017) Reliable partial ambiguity resolution for single-frequency GPS/BDS and INS integration. GPS Solut 21:251-264

Henderson HV, Searle SR (1981) On deriving the inverse of a sum of matrices. SIAM Rev 23:53-60

Julier SJ, Uhlmann JK, Durrant-Whyte HF (1995) A new approach for filtering nonlinear systems. In: Proceedings of the ACC'95, pp 1628-1632. doi: 10.1109/ACC.1995.529783

Li BF, Shen YZ, Li WX (2012) The seamless model for three-dimensional datum transformation. Sci China 55:2099-2108

Li ZK, Chang GB, Gao JX, Wang J, Hernandez A (2016) GPS/UWB/MEMS-IMU tightly coupled navigation with improved robust Kalman filter. Adv Space Res 58:2424-2434

Liu JN (1983) The equivalence of coordinate transformation models for the combination of satellite and terrestrial networks. J Wuhan Techn Univ Surv Mapp 8:37-50 (in Chinese with English abstract)

Liu J, Chen R (1998) Sequential Monte Carlo methods for dynamic systems. J Am Stat Assoc 93:1032-1044

Liu JN, Liu DJ (1985) The influence of the accuracy in geodetic and geocentric coordinates on combined adjustment. Acta Geod Cartogr Sin 14:133-144 (in Chinese with English abstract)

Liu JN, Liu DJ, Cui XZ (1987) Theory and applications of combined adjustment of satellite and terrestrial networks. J Wuhan Techn Univ Surv Mapp 12(4):1-9 (in Chinese with English abstract)

Lu J, Chen Y, Li BF, Fang X (2014) Robust total least squares with reweighting iteration for threedimensional similarity transformation. Surv Rev 46:28-36

Mahboub V (2012) On weighted total least-squares for geodetic transformation. J Geod 86:359-367

Mahboub V, Sharifi MA (2013) On weighted total least-squares with linear and quadratic constraints. J Geod 87:279-286

Mahboub V, Ardalan AA, Ebrahimzadeh S (2015) Adjustment of non-typical errors-invariables models. Acta Geod Geophys 50:207-218

Mahboub V, Saadatseresht M, Ardalan AA (2016) A general weighted total Kalman filter algorithm with numerical evaluation. Stud Geophys Geod 61:19-34

Mahboub V, Saadatseresht M, Ardalan AA (2017a) A solution to dynamic errors-invariables within system equations. Acta Geod Geophys. doi:10.1007/s40328-017-0201-0

Mahboub V, Saadatseresht M, Ardalan AA (2017b) On constrained integrated total Kalman filter for integrated direct geo-referencing. Surv Rev. doi:10.1080/00396265.2017.1341736 
Merwe R, Wan E (2001) The square-root unscented Kalman filter for state and parameter-estimation. Proc IEEE Int Conf Acoust Speech Signal Process (ICASSP) 6:3461-3464

Neitzel F (2010) Generalization of total least-squares on example of unweighted and weighted 2D similarity transformation. J Geod 84:751-762

Sage AP, Husa GW (1969) Adaptive filtering with unknown prior statistics. Joint Am Control Conf. doi:10. 1109/JACC.1969.4169325

Schaffrin B (2009) TLS collocation: the total least squares approach to EIV-models with stochastic prior information. In: 18th international, workshop on matrices and statistics, Solvakia

Schaffrin B, Iz HB (2008) Towards total Kalman filtering for mobile mapping. Intl Arch Photogr Rem Sens Spat Inf Sci 36:270-275

Schaffrin B, Uzun S (2011) Errors-In-Variables for mobile mapping algorithms. In: Thepresence of OutIIERS, archives of photogrammetry, cartography and remote sensing, vol 22. pp 377-387

Schaffrin B, Wieser A (2008) On weighted total least squares adjustment for linear regression. J Geod 82:415-421

Shen YZ, Li BF, Chen Y (2011) An iterative solution of weighted total least-squares adjustment. J Geod $85: 229-238$

Shi Y, Xu PL, Liu JN, Shi C (2015) Alternative formulae for parameter estimation in partial errors-invariables models. J Geod 89:13-16

Teunissen PJG (1988) The nonlinear 2D symmetric Helmert transformation: an exact nonlinear leastsquares solution. J Geod 62:1-15

Wang LY, Xu GY (2016) Variance component estimation for partial errors-in-variables models. Stud Geophys Geod 60:35-55

Wang LY, Zhao YW (2017) Unscented transformation with scaled symmetric sampling strategy for precision estimation of total least squares. Stud Geophys Geod. doi:10.1007/s11200-11016-11113-11200

Wang B, Li JC, Liu C (2016) A robust weighted total least squares algorithm and its geodetic applications. Stud Geophys Geod 60:177-194

Wang B, Li JC, Liu C, Yu J (2017) Generalized total least squares prediction algorithm for universal 3D similarity transformation. Adv Space Res 59(3):815-823

Xu GC (2003) GPS-theory, algorithms and applications, 2nd edn. Springer, Berlin

Xu PL (2016) The effect of errors-in-variables on variance component estimation. J Geod 90(8):681-701

Xu PL, Liu JN (2013) Variance components in errors-in-variables models: estimability, stability and bias analysis. In: Invited talk, VIII Hotine-Marussi symposium on mathematical geodesy, Rome, 17-21 June 2013

Xu PL, Liu JN (2014) Variance components in errors-in-variables models: estimability, stability and bias analysis. J Geod 88:719-734

Xu PL, Liu JN, Shi C (2012) Total least squares adjustment in partial errors-in-variables models: algorithm and statistical analysis. J Geod 86:661-675

Zhou YJ, Kou XJ, Zhu JJ, LI J (2014) A newton algorithm for weighted total least-squares solution to a specific errors-in-variables model with correlated measurements. Stud Geophys Geod 58:349-375 